\title{
REAÇÕES MULTICOMPONENTES DE BIGINELLI E DE MANNICH NAS AULAS DE QUÍMICA ORGÂNICA EXPERIMENTAL. UMA ABORDAGEM DIDÁTICA DE CONCEITOS DA QUÍMICA VERDE
}

\author{
Marcelo Volpatto Marques, Tula Beck Bisol e Marcus Mandolesi Sá* \\ Departamento de Química, Universidade Federal de Santa Catarina, 88040-900 Florianópolis - SC, Brasil
}

Recebido em 6/1/12; aceito em 26/3/12; publicado na web em 3/7/12

\begin{abstract}
BIGINELLI AND MANNICH MULTICOMPONENT REACTIONS IN THE UNDERGRADUATE ORGANIC CHEMISTRY LABORATORY. A DIDACTIC APPROACH TO GREEN CHEMISTRY PRINCIPLES. The introduction of Mannich and Biginelli multicomponent reactions in a practical Organic Chemistry course is presented in this article. Procedures described in the literature were adapted for use under the simple conditions available in undergraduate laboratories and were selected on the basis of Green Chemistry principles and practicality of synthesis. The reactions are easy to carry out and all products are readily isolated as crystalline solids with yields ranging from moderate to high.
\end{abstract}

Keywords: Mannich reaction; Biginelli reaction; Green Chemistry.

\section{INTRODUÇÃO}

A busca pelo desenvolvimento de processos químicos eficientes e ambientalmente seguros vem tornando a Síntese Orgânica uma prática cada vez mais sustentável e em conformidade com alguns dos princípios da Química Verde. ${ }^{1-5} \mathrm{Um}$ destes conceitos diz respeito à economia atômica, uma grandeza que mede a eficiência de uma transformação e é expressa pela quantidade relativa de átomos dos reagentes que são incorporados ao produto segundo a estequiometria da reação. ${ }^{6,7}$

Reações multicomponentes (RMCs) são transformações químicas em que três ou mais reagentes têm seus átomos incorporados ao produto final em uma única operação. ${ }^{8}$ As RMCs geralmente possuem excelente economia atômica e alguns exemplos importantes incluem as reações de Passerini, Ugi, Mannich e Biginelli. ${ }^{9-11}$

A reação de Biginelli é uma condensação tricomponente que envolve um composto 1,3-dicarbonilado, ureia (ou tioureia) e um aldeído na presença de um ácido de Brønsted como catalisador, fornecendo como produto a 3,4-di-hidropirimidin-2-ona multissubstituída 1 (Esquema 1). Diversas variações no procedimento original têm sido sugeridas, pois a reação requer aquecimento à alta temperatura, demanda longo tempo e leva ao produto esperado em baixo rendimento. Algumas dessas modificações incluem o uso de ácido de Lewis como catalisador, a ausência de solventes, a utilização de micro-ondas e, mais recentemente, o emprego de condições básicas. ${ }^{12,13} \mathrm{O}$ esforço para o desenvolvimento de metodologias mais eficientes para a síntese de $\mathbf{1}$ deve-se ao fato desta classe de compostos exibir um largo espectro de atividade biológica, incluindo antitumoral, antiviral, antibacteriana e anti-inflamatória, além de ser moduladora do canal de cálcio. ${ }^{14,15}$

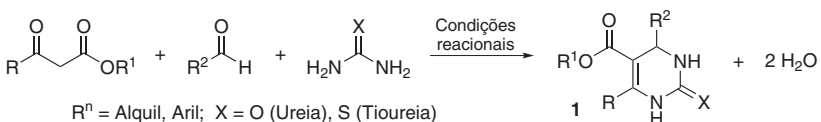

Esquema 1. Síntese de di-hidropirimidinonas 1 a partir da reação de Biginelli

Na reação de Mannich, a condensação tricomponente de uma cetona enolisável (contendo hidrogênio ácido $\alpha$ à carbonila), formaldeído e amônia, geralmente em meio ácido, fornece como produto

\footnotetext{
*e-mail: marcus.sa@ufsc.br
}

um composto $\beta$-amino carbonilado 2 (Esquema 2). Além de utilizar sais de amônio como fonte de nitrogênio, a reação de Mannich pode ser conduzida com aminas primárias ou secundárias, ou mesmo com amidas, na presença de um ácido apropriado. Compostos $\beta$-amino carbonílicos 2 obtidos pela reação de Mannich são blocos de construção versáteis em síntese orgância e podem ser convertidos em uma variedade de derivados úteis, incluindo aceptores de Michael e amino alcoóis, além de serem intermediários avançados na síntese de heterociclos nitrogenados e produtos naturais. ${ }^{16-18}$

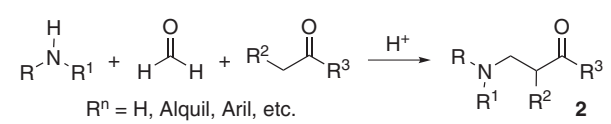

Esquema 2. Sintese de $\beta$-amino cetonas 2 a partir da reação de Mannich

Devido ao aumento do interesse na preparação de grandes quimiotecas de compostos nitrogenados, o aperfeiçoamento das RMCs tradicionais e o desenvolvimento de novas RMCs continuam sendo desafios para grupos de pesquisa acadêmicos e de corporações industriais. ${ }^{8}$ Portanto, estes tópicos devem estar presentes nos conteúdos programáticos de disciplinas de Química Orgânica.

A aplicação de RMCs em aulas experimentais de graduação tem sido pouco relatada na literatura, embora bons exemplos possam ser citados. ${ }^{19-23}$ Um deles envolve a reação de Mannich com enfoque no emprego de líquidos iônicos como solvente. ${ }^{21}$ Já a reação de Biginelli foi abordada por uma metodologia convencional, utilizando $\mathrm{HCl}$ como catalisador, ${ }^{22}$ ou com o emprego de ácido de Lewis na ausência de solvente. ${ }^{23}$

Neste trabalho, são apresentadas propostas de aulas experimentais em que os alunos elaboram e desenvolvem miniprojetos visando a obtenção dos produtos das reações multicomponentes de Biginelli e Mannich. O desenvolvimento dos miniprojetos inclui etapas de revisão bibliográfica, seleção de métodos com base em alguns dos princípios de Química Verde, na facilidade de execução e na acessibilidade de reagentes e, por fim, discussão dos resultados obtidos por cada equipe. Este processo permite uma comparação direta e instrutiva entre os métodos de síntese antigos e aqueles mais recentes, a partir de discussões envolvendo tópicos como economia atômica, custo dos reagentes e consumo de energia. Os produtos de cada transformação são sólidos cristalinos facilmente purificados por recristalização e caracterizados por ponto de fusão. Além disso, outros métodos de 
análise, como espectroscopia de infravermelho (IV) e ressonância magnética nuclear de hidrogênio $\left(\mathrm{RMN}{ }^{1} \mathrm{H}\right)$, também podem ser utilizados. Em suma, a atividade proposta envolve várias técnicas experimentais ensinadas em aulas práticas de Química Orgânica, entre elas o aquecimento sob refluxo, a extração com solventes, a cromatografia em coluna, a recristalização e a identificação dos compostos sintetizados. A seguir são apresentados os resultados obtidos em aulas experimentais de Química Orgânica para cada uma das duas RMCs.

\section{RESULTADOS E DISCUSSÃO}

\section{Reação de Biginelli}

Uma grande variedade de metodologias tem sido reportada visando aumentar a eficiência da reação de Biginelli em relação às condições convencionais, que utilizam ácidos minerais como catalisador. ${ }^{24}$ Entretanto, muitas transformações são lentas ou fazem uso de reagentes caros, meio fortemente ácido, grande volume de solventes ou quantidades estequiométricas de catalisadores, nem sempre disponíveis comercialmente. ${ }^{25-30}$ De modo a contornar estes obstáculos, as metodologias a serem trabalhadas em aulas experimentais devem empregar condições brandas, tempos de reação curtos (não superiores a 4 h) e reagentes comerciais a um custo acessível, preferencialmente sem o envolvimento de solventes orgânicos em quantidades excessivas. Diversas condições reacionais descritas na literatura para a síntese das di-hidropirimidin-2-onas multissubstituídas 6a-c (Esquema 3) a partir de acetoacetato de etila (3), aldeídos aromáticos 4 e ureia (5) possuem estas características e podem ser previamente apresentadas pelo professor, ${ }^{31-39}$ embora os alunos também sejam estimulados a buscar métodos alternativos na literatura.
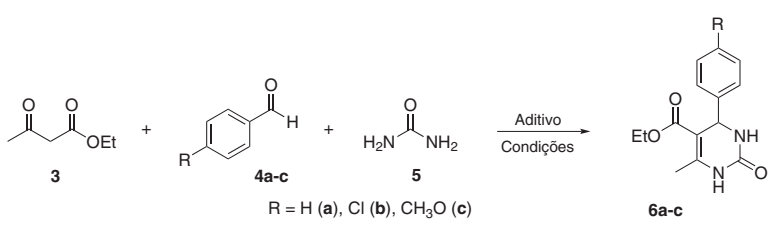

Esquema 3. Reações de Biginelli realizadas em aula

Após as etapas de pesquisa bibliográfica e discussão, foram selecionadas metodologias que utilizam aditivos simples, como os ácidos bórico, ${ }^{32}$ cloroacético ${ }^{33}$ ou para-toluenossulfônico, ${ }^{34}$ sais de amônio $\left(\mathrm{NH}_{4} \mathrm{Cl}\right),{ }^{35}$ lítio $(\mathrm{LiBr})^{36}$ ou cobre $\left(\mathrm{CuCl}_{2} \cdot 2 \mathrm{H}_{2} \mathrm{O}\right),{ }^{37}$ um agente oxidante (Oxone ${ }^{\circledR}: 382 \mathrm{KHSO}_{5} \cdot \mathrm{KHSO}_{4} \cdot \mathrm{K}_{2} \mathrm{SO}_{4}$ ) ou ainda condições na ausência de qualquer aditivo. ${ }^{39}$ As reações foram conduzidas, preferencialmente, na ausência de solvente e sob aquecimento durante um período de 1-3 h, dependendo da condição reproduzida (ver Parte Experimental).

Após um número expressivo de experimentos variando-se os aldeídos utilizados (benzaldeído, 4-clorobenzaldeído e, em menor grau, anisaldeído) é possível inferir algumas tendências e apontar os resultados que apresentaram maior reprodutibilidade. Por exemplo, nas reações para obtenção da di-hidropirimidin-2-ona 6a partindo de benzaldeído (4a), bons resultados foram observados com $\mathrm{NH}_{4} \mathrm{Cl}$, um aditivo barato e facilmente disponível..$^{35}$ Além disso, a condição livre de solvente torna atraente a aplicação deste procedimento em um laboratório de ensino. Já as preparações das di-hidropirimidin-2-onas $\mathbf{6 b}$ e $\mathbf{6 c}$ a partir dos respectivos aldeídos $\mathbf{4 b}$ e $\mathbf{4 c}$ foram alcançadas através de diversas metodologias, tanto pela combinação entre um aditivo e um solvente orgânico (p.ex., $\mathrm{H}_{3} \mathrm{BO}_{3}$ em $\mathrm{AcOH}^{32}$ ou $\mathrm{LiBr}$ em $\mathrm{CH}_{3} \mathrm{CN}^{36}$ ) como sob condições na ausência de solvente (empregando $\mathrm{ClCH}_{2} \mathrm{CO}_{2} \mathrm{H}^{33}$ ou $\mathrm{CuCl}_{2} \cdot 2 \mathrm{H}_{2} \mathrm{O}^{37}$ como aditivo).

Questões teóricas relativas à reação de Biginelli, incluindo os aspectos mecanísticos nas etapas de adição à carbonila e formação de ligação carbono-carbono, são conteúdos que podem ser explorados pelo professor. Porém, é importante destacar que os resultados observados aqui não permitem estabelecer uma relação direta entre a eficiência da reação de Biginelli e o efeito eletrônico do substituinte no aldeído. De fato, o objetivo da abordagem é discutir as diferentes metodologias sintéticas descritas na literatura, de modo a ressaltar as similaridades entre elas (por exemplo, nos mecanismos de reação fundamentados em catálise ácido-base) bem como suas diferenças, tanto nos aspectos químicos (reatividade, velocidade de reação) quanto econômicos e ambientais (custo dos reagentes e sustentabilidade).

Todas as condições reacionais empregadas neste estudo ${ }^{32-39}$ apresentaram facilidade de realização, tempos curtos de reação (3-4 h) e uso de reagentes comuns de laboratório. Os alunos confirmaram a obtenção dos produtos 6a-c por meio da medida do ponto de fusão e pela interpretação de seus espectros de IV (os dados espectrais obtidos estão apresentados na Parte Experimental e no Material Suplementar). Dessa forma, a reação de Biginelli pode ser convenientemente realizada em uma única aula experimental ou, opcionalmente, pode ser integrada ao experimento envolvendo a reação de Mannich.

\section{Reação de Mannich}

A reação de Mannich empregando aminas tornou-se um método bem estabelecido na literatura para a obtenção de compostos $\beta$-amino carbonilados 2 (Esquema 2). ${ }^{17,18}$ Entretanto, o uso de acetamida (9) nestas transformações visando a preparação dos respectivos compostos $\beta$-acetamido carbonílicos 7 e 8 é pouco relatado (Esquema 4). ${ }^{40-42}$ A dificuldade no emprego de amidas em reações de Mannich é associada, em parte, à sua menor reatividade quando comparada a aminas. Entre as condições reacionais recentemente desenvolvidas para viabilizar o acesso a compostos $\beta$-acetamidos carbonílicos 7 e $\mathbf{8}$, poucas são eficientes em promover reações sob tempos curtos, além de geralmente fazer uso de reagentes de alto custo ou comercialmente indisponíveis. ${ }^{40,41}$

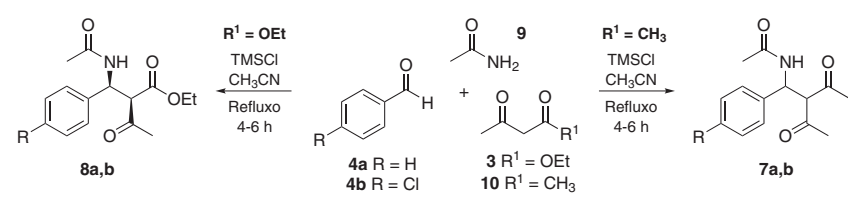

Esquema 4. Reações de Mannich realizadas em aula

Devido às restrições inerentes ao tempo de duração de uma aula experimental de Química Orgânica, muitas vezes são necessárias modificações dos procedimentos reacionais descritos na literatura para que as etapas de síntese, isolamento e caracterização de produtos possam ser executadas pelos alunos em períodos curtos (3-4 h). Recentemente, foi publicada ${ }^{42}$ a preparação dos compostos $\beta$-acetamido carbonílicos 7 e 8 a partir dos aldeídos $\mathbf{4 a}$ ou $\mathbf{4 b}$, acetamida (9), e $\beta$-cetoéster 3 ou 1,3-dicetona $\mathbf{1 0}$ em acetonitrila na presença de cloreto de trimetilsilano (TMSCl), que apesar de ser utilizado em quantidades equivalentes é um reagente disponível comercialmente, relativamente barato e normalmente presente em laboratórios de química devido a sua ampla utilização em transformações sintéticas ${ }^{43}$ (Esquema 4). Embora a reação forneça os produtos 7 e 8 em bons rendimentos, ${ }^{42}$ a necessidade de aquecimento sob refluxo por $5-10 \mathrm{~h}$ é, sem dúvida, um entrave à realização desta metodologia em aulas experimentais. Sendo assim, as condições reacionais foram adaptadas de modo a permitir a sua realização durante um período de 4-6 h, dividido em duas aulas (ver Parte Experimental).

Estas condições mostraram-se satisfatórias para a maioria dos casos envolvendo a preparação das $\beta$-acetamido dicetonas $\mathbf{7 a}, \mathbf{b}$ oriundas das reações entre a 1,3-dicetona $\mathbf{1 0}$, um aldeído (4a ou $\mathbf{4 b}$ ) 
e acetamida (9). Nestes casos, os produtos de Mannich obtidos pelos alunos apresentaram alta pureza e rendimentos de moderados a bons (ver Parte Experimental). Entretanto, quando se alterou o composto 1,3-dicarbonilado para acetoacetato de etila (3), a formação dos correspondentes $\beta$-acetamido cetoésteres $\mathbf{8}$ não se mostrou tão efetiva e somente o cetoéster $8 \mathbf{a}$ pôde ser obtido como um sólido possuindo ponto de fusão definido, apesar dos baixos rendimentos.

Apesar de terem sido conduzidas em duas aulas distintas, as reações foram reprodutíveis, de fácil execução e os experimentos apresentaram pouca influência pela alteração na quantidade de substrato utilizado (1-7 mmol), com os produtos sendo obtidos a partir de reagentes de laboratório comuns e baratos e dentro de condições encontradas em uma aula prática de graduação. Os alunos confirmaram a obtenção dos produtos $\mathbf{7 a}, \mathbf{7 b}$, e $\mathbf{8 a}$ por meio da medida do ponto de fusão e pela interpretação de seus espectros de IV (apresentados na Parte Experimental e no Material Suplementar).

Os experimentos envolvendo a reação de Mannich são realizados em duas aulas de $4 \mathrm{~h}$ e podem ser convenientemente integrados à reação de Biginelli, ou seja, enquanto ocorre a reação de Mannich (aquecimento sob refluxo durante duas aulas, no total de 4-6 h) os alunos podem executar até duas reações de Biginelli, no total de três aulas de 3-4 h (a última para tratamento e purificação por recristalização). Portanto, as práticas aqui descritas são modulares e podem ser desenvolvidas em diversas combinações, sendo que o professor opta pela aplicação de uma ou mais RMCs, dependendo dos recursos disponíveis no laboratório e das particularidades de cada curso. Finalmente, é importante enfatizar que em todos os experimentos os alunos foram responsáveis por escrever relatórios com discussões e interpretações completas de seus resultados, além de incluir o uso de pesquisa bibliográfica para suportar seus argumentos.

\section{CONCLUSÃO}

Neste trabalho, os protocolos selecionados na literatura para a obtenção dos produtos de Biginelli 6a-c e Mannich 7a-b e 8a, empregando condições brandas e reagentes disponíveis no laboratório, foram reproduzidos pelos alunos com as devidas adaptações. Os estudantes foram confrontados com a necessidade de reconhecer alguns dos princípios da Química Verde em uma reação química, com enfoque especial em eficiência catalítica e economia atômica. Os principais aspectos pedagógicos abordados incluíram pesquisa bibliográfica, seleção de métodos, execução dos experimentos e comparação dos resultados obtidos, bem como reforço dos conteúdos teóricos dos cursos básicos de Química Orgânica e dos princípios da Química Verde. Finalmente, conceitos inerentes às transformações químicas trabalhadas, como reações de adição à carbonila, formação de ligação carbono-carbono e efeitos estereoeletrônicos, também podem ser explorados nas aulas experimentais.

\section{PARTE EXPERIMENTAL}

\section{Reação de Biginelli - preparação geral}

Como as reações foram reproduzidas conforme as diferentes metodologias reportadas na literatura, a seguir apresenta-se um procedimento geral. Deste modo, as quantidades de reagentes, o tipo de aditivo, ${ }^{44}$ o uso de solvente, o emprego de aquecimento, o tempo reacional e a forma de tratamento da reação, bem como o processo de recristalização, são dependentes da referência bibliográfica utilizada (dados mais específicos contendo tipo e quantidade do aditivo empregado, solvente, tempo de reação, temperatura e rendimento obtido para alguns exemplos selecionados estão apresentados no Material Suplementar).
Em um balão de fundo redondo foram introduzidos 1 equiv. do aldeído, 1 equiv. de acetoacetato de etila, 1 equiv. de ureia e, quando foi o caso, o solvente e o aditivo nas quantidades descritas. As reações foram mantidas sob agitação magnética à temperatura ambiente ou sob aquecimento nos tempos determinados na literatura. Nas reações sob aquecimento foi adaptado um condensador à boca do balão e o sistema foi aquecido com banho de água (até 70-80 ${ }^{\circ} \mathrm{C}$ ) ou areia (acima de $80^{\circ} \mathrm{C}$ ) nas temperaturas reportadas. As misturas reacionais foram submetidas a tratamentos que, na maioria das vezes, consistiram de adição de água gelada e filtração do produto sólido formado. Nos casos onde a precipitação em água não ocorreu, a mistura aquosa foi transferida para um funil de separação e extraída com 2-3 porções de diclorometano. As fases orgânicas combinadas foram secas sobre $\mathrm{Na}_{2} \mathrm{SO}_{4}$ anidro, filtradas e o solvente foi evaporado. O sólido obtido foi purificado por recristalização, geralmente empregando etanol, para fornecer como produto a di-hidropirimidin-2-ona $\mathbf{6}$ com alto grau de pureza.

\section{5-Etoxicarbonil-4-fenil-6-metil-3,4-di-hidropirimidin-2-ona (6a)}

Rendimento: $15-90 \%$. Ponto de fusão médio: $205-208{ }^{\circ} \mathrm{C}$ (Lit. ${ }^{37}$ 208-209 ${ }^{\circ} \mathrm{C}$ ). IV (KBr): $v_{\max } 3245,3116,2979,2939,1727,1702$, $1649,1465,1421,1314,1290,1222,1091,780 \mathrm{~cm}^{-1}$.

\section{4-(4-Clorofenil)-5-etoxicarbonil-6-metil-3,4-di-hidropirimidin-2- ona $(\boldsymbol{6} \boldsymbol{b})$}

Rendimento: $20-85 \%$. Ponto de fusão médio: $212-215{ }^{\circ} \mathrm{C}$ (Lit. ${ }^{37}$ 215-216 $\left.{ }^{\circ} \mathrm{C}\right)$. IV (KBr): $v_{\max } 3240,3118,2981,2957,1724,1707$, $1650,1462,1290,1220,1089,781 \mathrm{~cm}^{-1}$.

\section{5-Etoxicarbonil-6-metil-4-(4-metoxifenil)-3,4-di-hidropirimidin-2- ona $(\boldsymbol{6 c})$ \\ Rendimento: 40-80\%. Ponto de fusão médio: $203-205{ }^{\circ} \mathrm{C}$ (Lit. ${ }^{37}$ 200-202 $\left.{ }^{\circ} \mathrm{C}\right)$. IV (KBr): $\vee_{\max } 3243,3112,2982,2957,1724,1706$, 1650, 1514, 1459, 1279, 1221, 1087, $791 \mathrm{~cm}^{-1}$.}

\section{Reação de Mannich}

Em um balão de fundo redondo foram introduzidos 5,0 mmol do aldeído (4a ou 4b), 6,0 mmol do composto 1,3-dicarbonilado (3 ou 10), 6,0 mmol de acetamida (9) e $15 \mathrm{~mL}$ de $\mathrm{CH}_{3} \mathrm{CN}$. A seguir, 5,0 mmol de TMSCl foram adicionados sob agitação magnética, o balão foi acoplado a um condensador e a uma fonte de aquecimento (banho de água ou areia) e a reação foi mantida sob agitação e refluxo por 2-3 h. Após resfriar a mistura, o sistema de refluxo foi desmontado e o balão reacional foi fechado e guardado à temperatura ambiente. $\mathrm{Na}$ aula seguinte, o aquecimento sob refluxo foi retomado e a reação deixada sob agitação por mais 2-3 h, totalizando um período de 4-6 h. Após o término da reação, $30 \mathrm{~mL}$ de água foram adicionados, a mistura foi extraída com EtOAc ( 3 × $20 \mathrm{~mL})$ e as fases orgânicas combinadas foram lavadas com água $(3 \times 20 \mathrm{~mL})$ e secas sobre $\mathrm{Na}_{2} \mathrm{SO}_{4}$ anidro. Após filtração, o solvente foi evaporado e o resíduo bruto obtido recristalizado em EtOAc/hexano, fornecendo a $\beta$-acetamido dicetona 7 ou o $\beta$-acetamido cetoéster 8 na forma de um sólido branco.

\section{3-Acetil-4-(N-acetilamino)-4-fenilbutan-2-ona (7a)}

Rendimento: $40-80 \%$. Ponto de fusão médio: $131-132{ }^{\circ} \mathrm{C}$ (Lit. ${ }^{42}$ $\left.132-134{ }^{\circ} \mathrm{C}\right)$. IV $(\mathrm{KBr}): v_{\max } 3359,2965,1700,1650,1529,1366$, $1200,758,706 \mathrm{~cm}^{-1}$.

\section{3-Acetil-4-(N-acetilamino)-4-(4-clorofenil)butan-2-ona (7b)}

Rendimento: $30-70 \%$. Ponto de fusão médio: $165-167{ }^{\circ} \mathrm{C}$ (Lit. ${ }^{42}$ $\left.167-169^{\circ} \mathrm{C}\right)$. IV (KBr): $v_{\max } 3291,1722,1702,1650,1538,1371$, $1291,1093,539 \mathrm{~cm}^{-1}$. 
2-Acetil-3-(N-acetilamino)-3-fenilpropanoato de etila $(\mathbf{8 a})$

Rendimento: 10-20\%. Ponto de fusão médio: $106-108{ }^{\circ} \mathrm{C}$ (Lit. ${ }^{42}$ 106-109 $\left.{ }^{\circ} \mathrm{C}\right)$. IV (KBr): $v_{\max } 3343,1733,1715,1652,1536,1371$, $1306,1150,705 \mathrm{~cm}^{-1}$.

\section{MATERIAL SUPLEMENTAR}

O material suplementar está disponível em http://quimicanova. sbq.org.br, na forma de arquivo PDF com acesso livre, e contém os espectros de IV dos compostos $\mathbf{6 a}, \mathbf{6 b}, \mathbf{6 c}, \mathbf{7 a}, 7 \mathbf{b}$ e $\mathbf{8 a}$.

\section{AGRADECIMENTOS}

Aos alunos dos cursos de Química que participaram das aulas experimentais, aos funcionários da Central de Análises e do Departamento de Química da UFSC, ao INCT-Catálise e às agências de fomento CAPES, CNPq e FAPESC pelas bolsas de estudo e pelo apoio financeiro.

\section{REFERÊNCIAS E NOTAS}

1. Anastas, P. T.; Tetrahedron 2010, 66, 1026.

2. Tucker, J. L.; Org. Process Res. Dev. 2010, 14, 328.

3. Sheldon, R. A.; Green Chem. 2007, 9, 1273.

4. Silva, F. M.; Lacerda, P. S. B.; Jones Jr., J.; Quim. Nova 2005, 28, 103.

5. Braun, B.; Charney, R.; Clarens, A.; Farrugia, J.; Kitchens, C.; Lisowski, C.; Naistat, D.; O'Neil, A.; J. Chem. Educ. 2006, 83, 1126.

6. Trost, B. M.; Acc. Chem. Res. 2002, 35, 695.

7. Merat, L. M. O. C.; Gil, R. A. S. S.; Quim. Nova 2003, 26, 779

8. Biggs-Houck, J. E.; Younai, A.; Shaw, J. T.; Curr. Opin. Chem. Biol. 2010, 14, 371 .

9. Dömling, A.; Chem. Rev. 2006, 106, 17.

10. Córdova, A.; Acc. Chem. Res. 2004, 37, 102.

11. Kappe, C. O.; Acc. Chem. Res. 2000, 33, 879.

12. Shen, Z.-L.; Xu, X.-P.; Ji, S.-J.; J. Org. Chem. 2010, 75, 1162.

13. Wan, J.-P.; Liu, Y.; Synthesis 2010, 3943.

14. Kappe, C. O.; Eur. J. Med. Chem. 2000, 35, 1043.

15. Russowsky, D.; Canto, R. F. S.; Sanches, S. A. A.; D’Oca, M. G. M.; Fátima, A.; Pilli, R. A.; Kohn, L. K.; Antônio, M. A.; Carvalho, J. E.; Bioorg. Chem. 2006, 34, 173.

16. Bur, S. K.; Martin, S. F.; Tetrahedron 2001, 57, 3221.

17. Sapi, J.; Laronze, J.-Y.; Arkivoc 2004, vii, 208.

18. Tu, S.-J.; Jiang, B.; Zhang, J.-Y.; Jia, R.-H.; Zhang, Y.; Yao, C.-S.; Org. Biomol. Chem. 2006, 4, 3980.

19. Hooper, M. M.; DeBoef, B.; J. Chem. Educ. 2009, 86, 1077.
20. Bossio, R.; Marcaccini, S.; Pepino, R.; Marcos, C. F.; J. Chem. Educ. 2000, 77, 382.

21. Mak, K. K. W.; Siu, J.; Lai, Y. M.; Chan, P.-K.; J. Chem. Educ. 2006, 83, 943.

22. Holden, M. S.; Crouch, R. D.; J. Chem. Educ. 2001, 78, 1104.

23. Aktoudianakis, E.; Chan, E.; Edward, A. R.; Jarosz, I.; Lee, V.; Mui, L.; Thatipamala, S. S.; Dicks, A. P.; J. Chem. Educ. 2009, 86, 730.

24. Kolosov, M. A.; Orlov, V. D.; Beloborodov, D. A.; Dotsenko, V. V.; Mol. Diversity 2009, 13, 5 .

25. Bigi, F.; Carloni, S.; Frullanti, B.; Maggi, R.; Sartori, G.; Tetrahedron Lett. 1999, 40, 3465.

26. Ma, Y.; Qian, C.; Wang, L.; Yang, M.; J. Org. Chem. 2000, 65, 3864.

27. Ranu, B. C.; Hajra, A.; Jana, U.; J. Org. Chem. 2000, 65, 6270.

28. Hu, E. H.; Sidler, D. R.; Dolling, U.-H.; J. Org. Chem. 1998, 63, 3454.

29. Li, J.-T.; Han, J.-F.; Yang, J.-H.; Li, T.-S.; Ultrason. Sonochem. 2003,10, 119.

30. Yarapathi, R. V.; Kurva, S.; Tammishetti, S.; Catal. Commun. 2004, 5, 511.

31. Chari, M. A.; Syamasundar, K.; J. Mol. Catal. A: Chem. 2004, 221, 137.

32. Tu, S.; Fang, F.; Miao, C.; Jiang, H.; Feng, Y.; Shi, D.; Wang, X.; Tetrahedron Lett. 2003, 44, 6153.

33. Yu, Y.; Liu, D.; Liu, C.; Luo, G.; Bioorg. Med. Chem. Lett. 2007, 17, 3508.

34. Bose, A. K.; Pednekar, S.; Ganguly, S. N.; Chakraborty, G.; Manhas, M. S.; Tetrahedron Lett. 2004, 45, 8351.

35. Shaabani, A.; Bazgir, A.; Teimouri, F.; Tetrahedron Lett. 2003, 44, 857.

36. Maiti, G.; Kundu, P.; Guin, C.; Tetrahedron Lett. 2003, 44, 2757.

37. Singh, O. M.; Singh, S. J.; Devi, M. B.; Devi, L. N.; Singh, N. I.; Lee, S.-G.; Bioorg. Med. Chem. Lett. 2008, 18, 6462.

38. Niknam, K.; Zolfigol, M. A.; Hossieninejad, Z.; Daneshvar, N.; Chin. J. Catal. 2007, 28, 591.

39. Ranu, B. C.; Hajra, A.; Dey, S. S.; Org. Process Res. Dev. 2002, 6, 817.

40. Shinu, V. S.; Sheeja, B.; Purushothaman, E.; Banulayan, D.; Tetrahedron Lett. 2009, 50, 4838.

41. Davoodnia, A.; Heravi, M. M.; Rezaei-Daghigh, L.; Tavakoli-Hoseini, N.; Chin. J. Chem. 2010, 28, 429.

42. Mao, H.; Wan, J.; Pan, Y.; Tetrahedron 2009, 65, 1026.

43. $100 \mathrm{~mL}$ de TMSCl (número do registro CAS 75-77-4) custam $€ 15,70$ (ACROS - número do produto 110121000; fonte: www.acros.com, acessada em 20/06/2012). Com $100 \mathrm{~mL}$ de TMSCl, cerca de 150 equipes poderiam realizar a reação de Mannich na escala de $5 \mathrm{mmol}$.

44. Embora sejam, em sua maioria, usados em pequenas quantidades nas reações, os resíduos de aditivos contendo metais no estado sólido ou em solução devem ser separados e identificados para tratamento e disposição final. 


\section{REAÇÕES MULTICOMPONENTES DE BIGINELLI E DE MANNICH NAS AULAS DE QUÍMICA ORGÂNICA EXPERIMENTAL. UMA ABORDAGEM DIDÁTICA DE CONCEITOS DA QUÍMICA VERDE}

Marcelo Volpatto Marques, Tula Beck Bisol e Marcus Mandolesi Sá*

Departamento de Química, Universidade Federal de Santa Catarina, 88040-900 Florianópolis - SC, Brasil

\section{CONSIDERAÇÕES GERAIS}

Os solventes e reagentes empregados nas sínteses e caracterizações foram adquiridos de fontes comerciais (Aldrich, Acros, Fluka, Nuclear, Synth) e utilizados sem prévia purificação. Os pontos de fusão das substâncias sólidas foram determinados em um aparelho Microquímica MQAPF-301 e não foram corrigidos. Os espectros no infravermelho foram registrados em um espectrofotômetro Perkin-Elmer Spectrum 100 FT-IR, com as frequências de absorção sendo expressas em $\mathrm{cm}^{-1}$. As amostras, todas sólidas, foram analisadas em pastilhas de $\mathrm{KBr}$.<smiles>[R]c1ccc(C2NC(=O)NC(C)=C2C(=O)OCC)cc1</smiles>

Tabela 1S. Exemplos selecionados da reação de Biginelli para a preparação de 3,4-di-hidropirimidin-2-onas 6a-c

\begin{tabular}{|c|c|c|c|c|c|c|}
\hline$\#$ & $\mathrm{R}$ & Aditivo (mol \%) & Solvente & $\mathrm{T}\left({ }^{\circ} \mathrm{C}\right)$ & Tempo (h) & Rend. $(\%)^{\mathrm{a}}$ \\
\hline $6 \mathbf{a}$ & $\mathrm{H}$ & $\operatorname{LiBr}(10)$ & $\mathrm{CH}_{3} \mathrm{CN}$ & Refluxo & 3 & 15 \\
\hline $6 \mathbf{a}$ & $\mathrm{H}$ & $\mathrm{NH}_{4} \mathrm{Cl}(40)$ & - & 100 & 3 & $45-75^{\mathrm{b}}$ \\
\hline $6 \mathbf{b}$ & $\mathrm{Cl}$ & $\operatorname{LiBr}(10)$ & $\mathrm{CH}_{3} \mathrm{CN}$ & Refluxo & 4 & $20-30^{\mathrm{b}}$ \\
\hline $6 \mathbf{b}$ & $\mathrm{Cl}$ & $\mathrm{CuCl}_{2} \cdot 2 \mathrm{H}_{2} \mathrm{O}(20)$ & - & 100 & 2 & $40-50^{\mathrm{b}}$ \\
\hline $6 \mathbf{b}$ & $\mathrm{Cl}$ & $\mathrm{ClCH}_{2} \mathrm{COOH}(10)$ & - & 90 & 2,5 & 35 \\
\hline $6 c$ & $\mathrm{CH}_{3} \mathrm{O}$ & $\mathrm{NH}_{4} \mathrm{Cl}(40)$ & - & 100 & 3 & 45 \\
\hline $6 c$ & $\mathrm{CH}_{3} \mathrm{O}$ & $\mathrm{H}_{3} \mathrm{BO}_{3}(20)$ & $\mathrm{CH}_{3} \mathrm{COOH}$ & 100 & 3 & 75 \\
\hline
\end{tabular}

${ }^{a}$ Rendimento do produto sólido obtido após recristalização. ${ }^{b}$ Faixa de rendimento observado para três experimentos distintos.

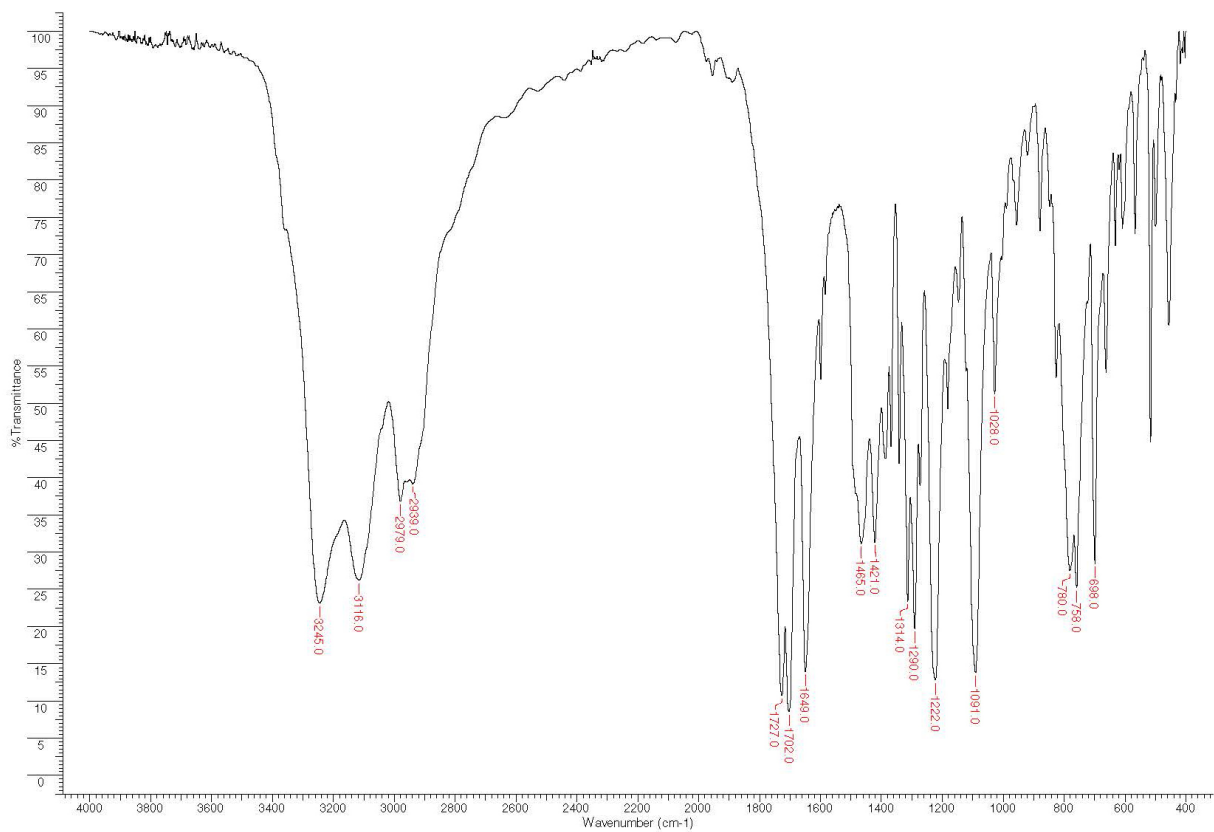

Figura 1S. $\mathrm{IV}\left(\mathrm{KBr}, \mathrm{cm}^{-1}\right)$ da di-hidropirimidinona $\mathbf{6 a}$

*e-mail: marcus.sa@ufsc.br 


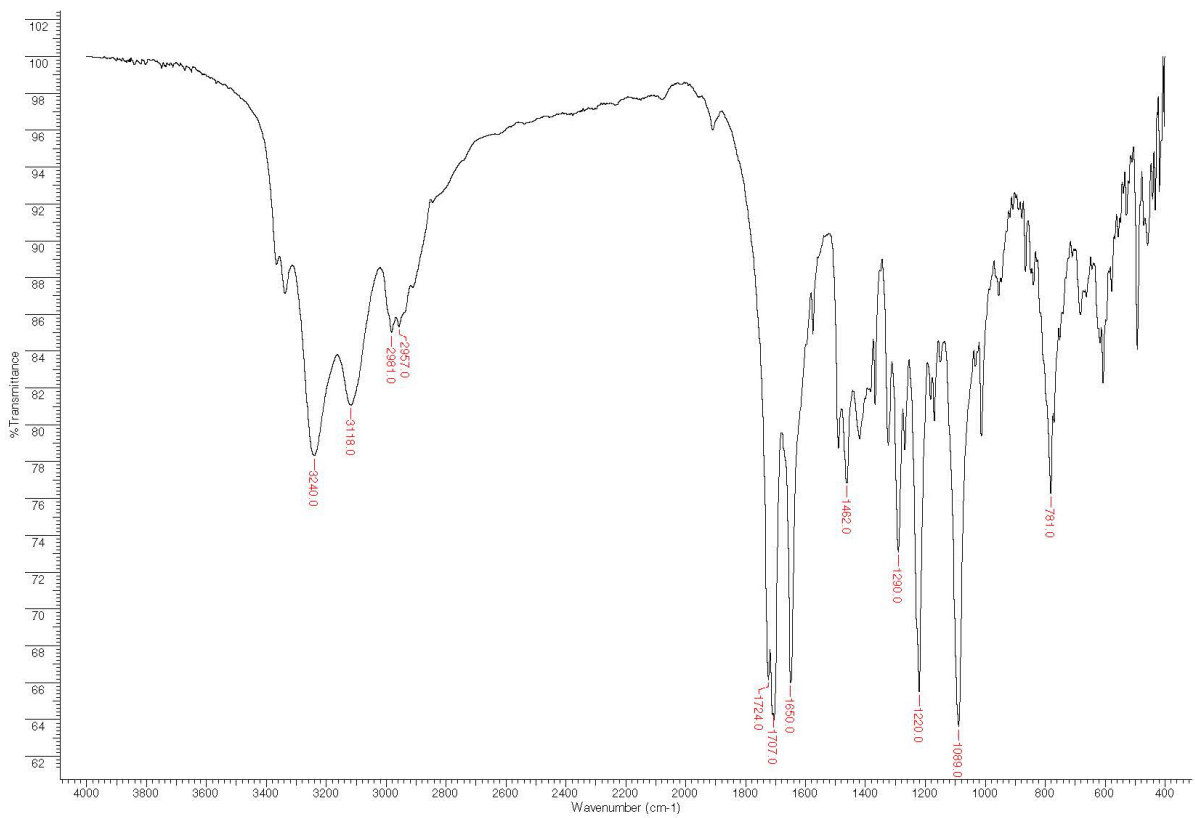

Figura $2 S . I V\left(K B r, \mathrm{~cm}^{-1}\right)$ da di-hidropirimidinona $\boldsymbol{6} \boldsymbol{b}$

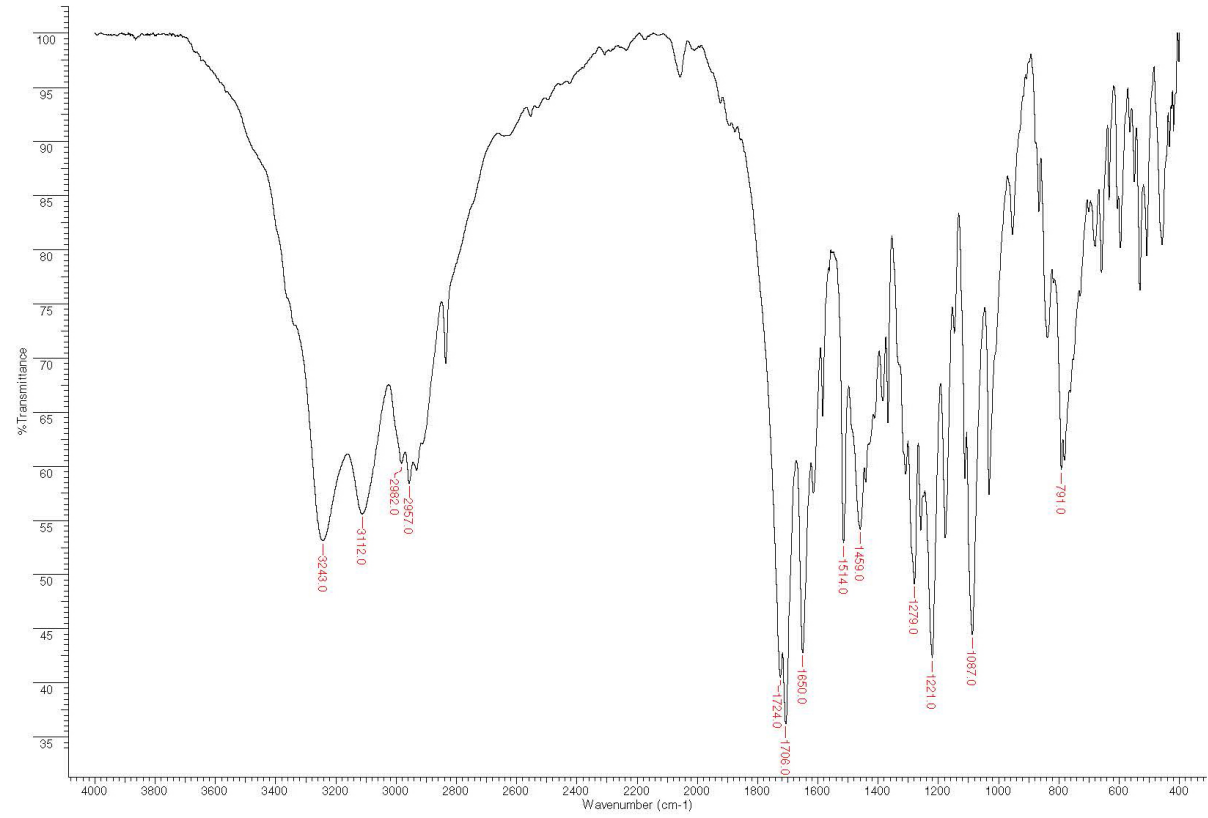

Figura 3S. $\mathrm{IV}\left(\mathrm{KBr}, \mathrm{cm}^{-1}\right)$ da di-hidropirimidinona $\mathbf{6 c}$ 


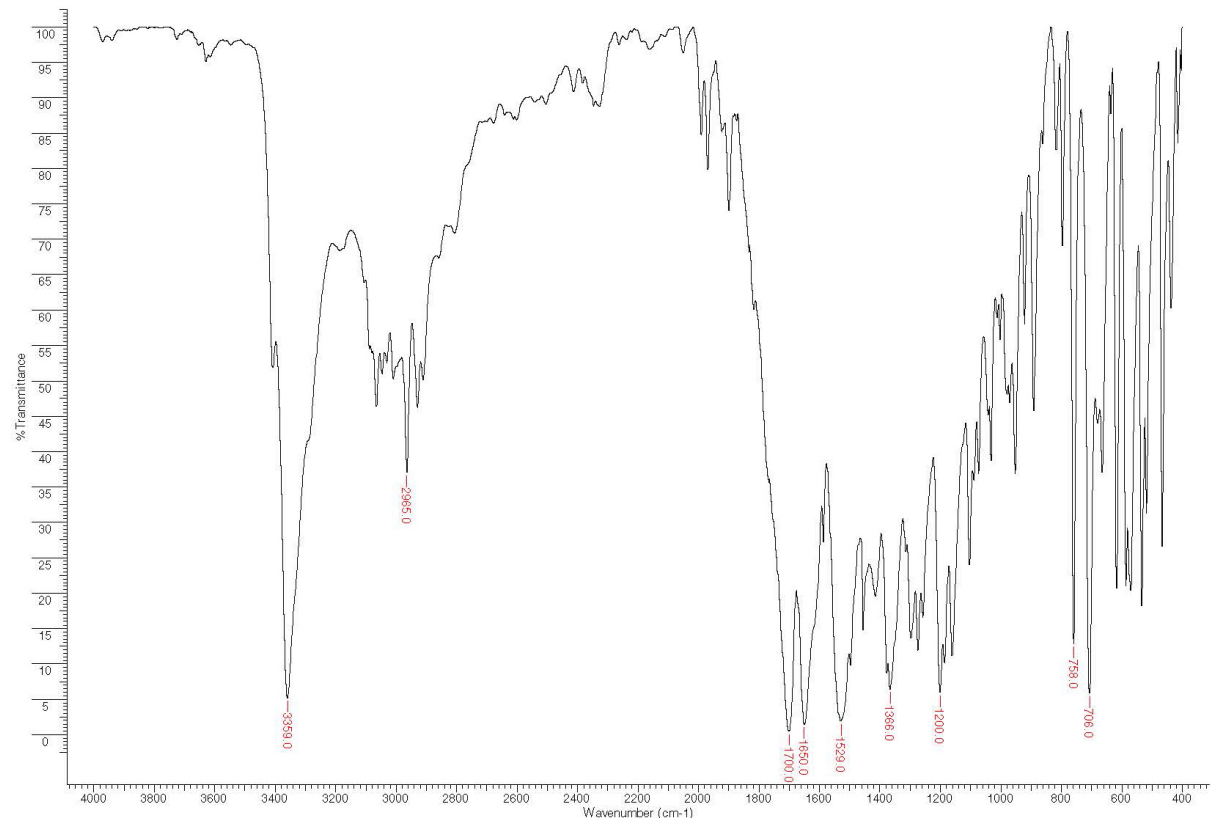

Figura 4S. IV $\left(\mathrm{KBr}, \mathrm{cm}^{-1}\right)$ da $\beta$-acetamido dicetona $7 a$

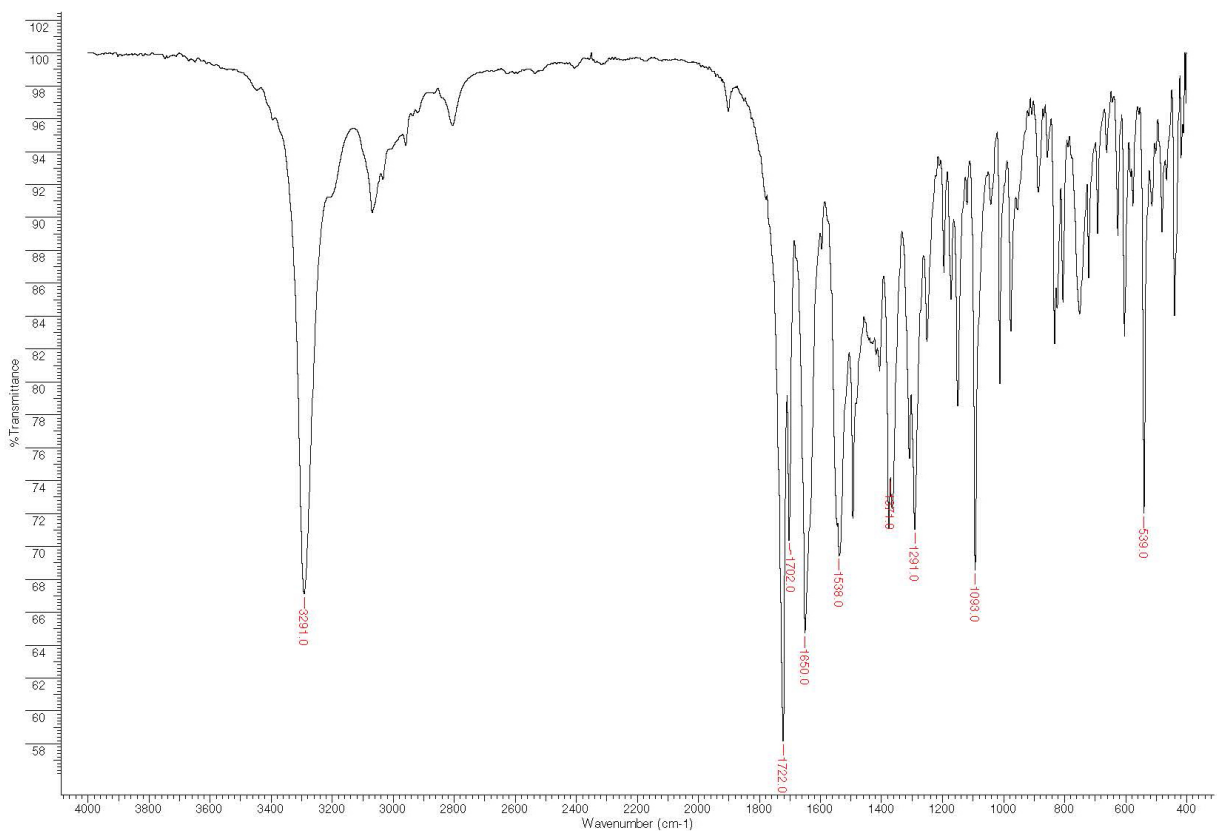

Figura 5S. IV $\left(\mathrm{KBr}, \mathrm{cm}^{-1}\right)$ da $\beta$-acetamido dicetona $7 \boldsymbol{b}$ 


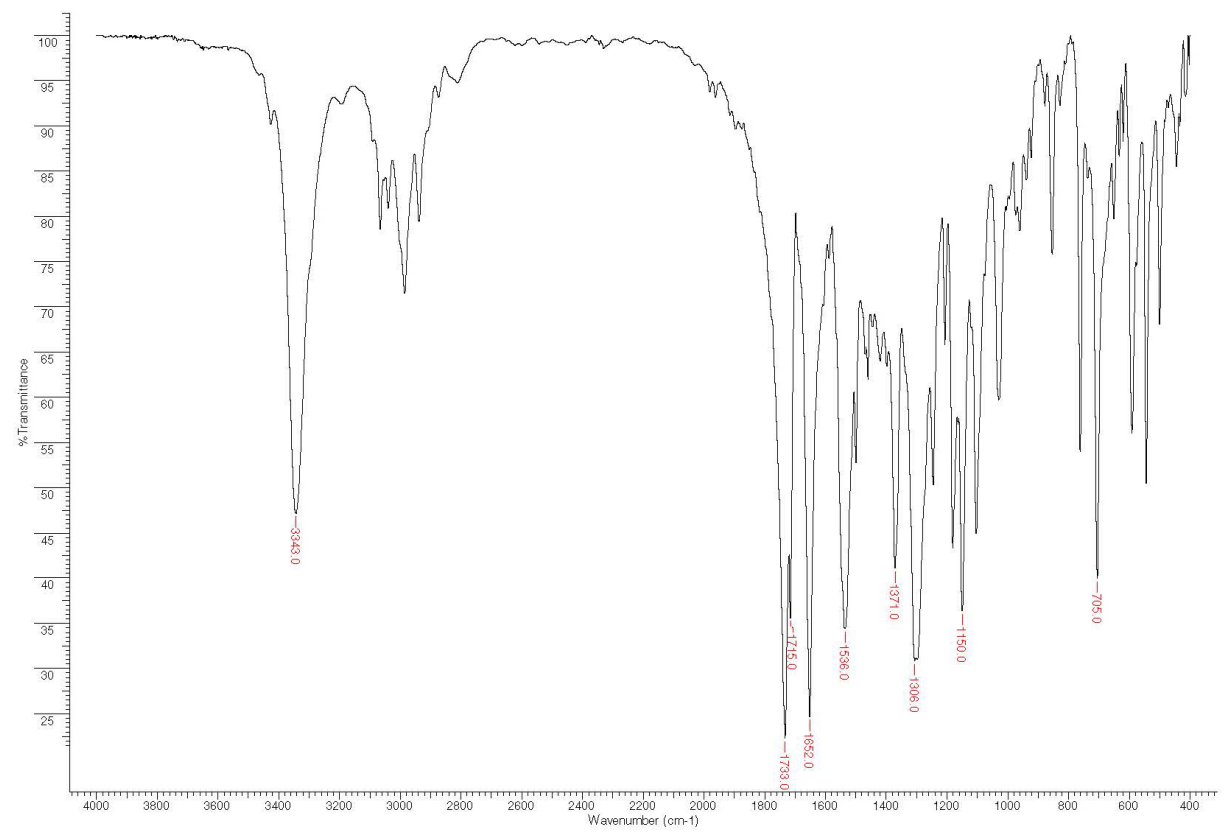

Figura 6S. IV $\left(\mathrm{KBr}, \mathrm{cm}^{-1}\right)$ do $\beta$-acetamido cetoéster $8 \boldsymbol{a}$ 